\title{
Experimental Characterization of Ultra-Wideband Channel Parameter Measurements in an Underground Mine
}

\author{
B. Nkakanou, G. Y. Delisle, and N. Hakem \\ LRTCS-UQAT, 450 3eme Avenue Local 105, Val-d'Or, QC, Canada J9P1S2 \\ Correspondence should be addressed to B. Nkakanou, bertrand.nkakanou@uqat.ca
}

Received 6 May 2011; Accepted 25 August 2011

Academic Editor: Mohamed El-Tanany

Copyright () 2011 B. Nkakanou et al. This is an open access article distributed under the Creative Commons Attribution License, which permits unrestricted use, distribution, and reproduction in any medium, provided the original work is properly cited.

Experimental results for an ultra-wideband (UWB) channel parameters in an underground mining environment over a frequency range of $3 \mathrm{GHz}$ to $10 \mathrm{GHz}$ are reported. The measurements were taken both in LOS and NLOS cases in two different size mine galleries. In the NLOS case, results were acquired for different corridor obstruction angles. The results were obtained during an extensive measurement campaign in the UWB frequency, and the measurement procedure allows both the large- and small-scale parameters such as the path loss exponent, coherence bandwidth, and so forth, to be quantified. The capacity of the UWB channel as a function of the physical depth of the mine gallery has also been recorded for comparison purposes.

\section{Introduction}

Ultra-wideband (UWB) radio is a technology that has attracted a great deal of interest from academia, industry, and global standardization bodies over the recent years. The FCCs are defined as UWB signals having $10 \mathrm{~dB}$ bandwidth greater than $25 \%$ of their center frequencies of the radiation [1].

Ultra-wideband is a wireless technology that has offered many advantages, mainly the high-speed data transmissions, short-duration pulse, low-power spectral density, and large instantaneous bandwidth. Theses specifications allow coexistence with other existing technologies and enable fine time resolution. Many studies have shown that UWB is a good candidate for short-range multiple-access communications in dense multipath environments [2-4]. The potential of UWB system provides the following features: multiple accesses due to wide transmission bandwidths, accurate position location and ranging, lack of significant multipath fading due to fine delay resolution, and protected communications due to low transmission power.

In order to establish a suitable model for the ultrawideband channel behavior which is used when designing WB communications systems to support applications in underground mine environment, it is then important to have a thorough understanding of the propagation channel constraints.
In recent years, several measurement campaigns to characterize the mining channel were conducted by the Télébec Underground Communications Research Laboratory (LRTCS) located in the mining area of Val-d'Or, QC, Canada $[5,6]$. This laboratory, specialized in complex confined area communication, has at its disposal a unique experimental mine (CANMET-Canadian Centre for Minerals and Energy Technology).

Characterization of channel propagation in underground environments has been an area of research since many years, but these studies have been carried out almost exclusively in tunnels. Also, many propagation measurements have been carried out for indoor narrowband propagation channels, and several models have been proposed in the literature [57]. However, due to their restricted measurement bandwidth, they were inappropriate for the UWB systems.

Similarly, UWB measurements and characterization of channels in indoor and outdoor environments [8-11] have been also performed in that type of environment. However, despite these various research efforts, there is still a significant lack of information about propagation characteristics of UWB systems in a confined environment.

The objectives of this paper are to present measurements and characterization of ultra-wideband (UWB) propagation in an underground mining environment and to detail the experimental procedures and measurement setup used to collect the data. Experimental procedures and locations 
where the measurements were carried out are reported with full detail; then, the postprocessing of the acquired data is explained. Finally, the results pertaining to the signal quality, small-scale effects, large-scale path loss exponents, time dispersion parameters, and capacity are discussed. Some sitespecific trends and observations are described, and channel performances for two types of directive and omnidirectional antennas are compared.

The paper is organized as follows. In Section 2, the mining environment and measurement setup are presented. In Section 3, relevant channel parameters are presented based on the measurements. Section 4 presents the conclusions.

\section{Measurement System}

Intensive measurements were carried out in underground galleries of a former gold mine at CANMET in Val-d'Or, QC, Canada. The measurements were conducted at two levels $(40 \mathrm{~m}$ and $70 \mathrm{~m})$ in the mine. The purpose of these measurements was to achieve a characterization of the two propagation channels based on statistical analysis and modelling of the channel's impulse response. The measurements were taken in both line of sight (LOS) and non-line of sight (NLOS) scenarios. The measurements were taken between $1 \mathrm{~m}$ and $10 \mathrm{~m}$ in LOS and in NLOS cases at regular interval of $1 \mathrm{~m}$. A description of measurement plan is described in Figure 1.

2.1. Measurement Plan. The goal of the measurements was to investigate the small- and large-scale variations in the statistics of the channel. Large-scale variations are due to high antenna separation changes, one meter, for instance, where small-scale variations indicate changes in statistics when the moving antenna position changes by a few centimeters. The environment mainly consists of very rough walls; the floor is uneven, and there are some puddles of water mainly at level 70. Figure 1 illustrates the map of the gallery with all its adjacent galleries at level 70 .

At level 70, the dimensions of the mine corridors are approximately $2.5 \mathrm{~m}$ in width and $3 \mathrm{~m}$ in height (Figure 2). The gallery walls are somewhat discontinuous, and the lengths of the galleries used for the experimentation are approximately 80 meters in length for level 70 and 100 meters at level 40. In NLOS case, measurements at different angles of obstruction were taken.

At level 40, the dimensions of the mine corridors are $4 \mathrm{~m}$ in width and approximately $5 \mathrm{~m}$ in height. This gallery is broader and higher than the one at level 70, and the floor slope is about 15 degrees.

2.2. Measurements. A vector network analyzer (VNA) is measurement equipment which can measure scattering parameters or S-parameters, which gives both the amplitude and phase of the reflection and/or transmission coefficients of the device under test, here the UWB channel. A communication channel can be viewed as a two-port passive device with input and output ports located at the transmitter and receiver, respectively. The advantage of using a VNA as the transceiver is its stepped frequency mode of operation and its

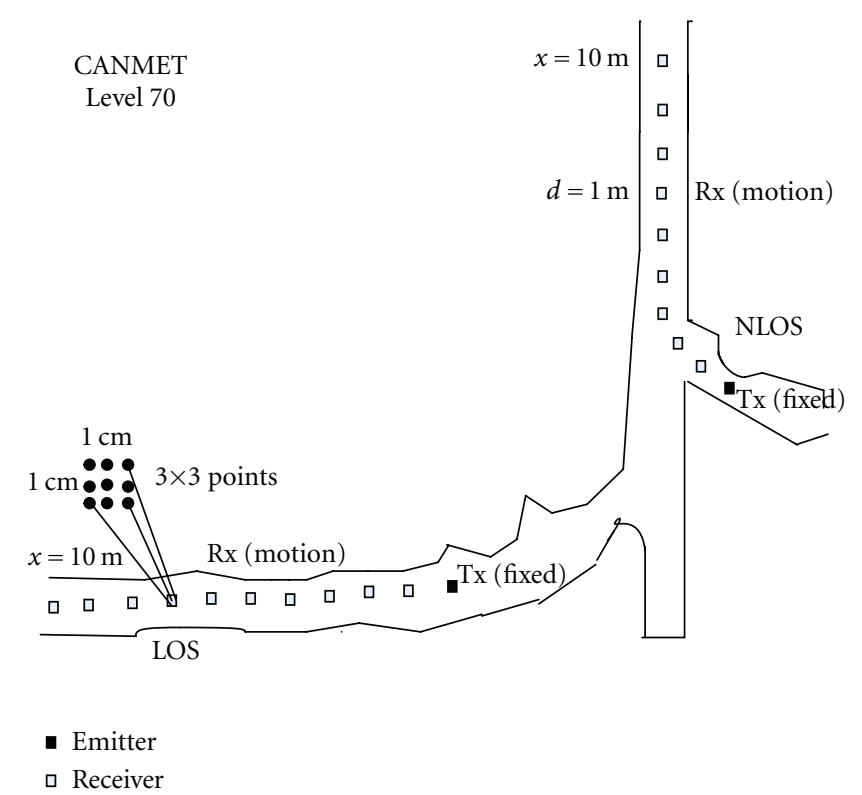

Figure 1: Map of the underground gallery at level 70.

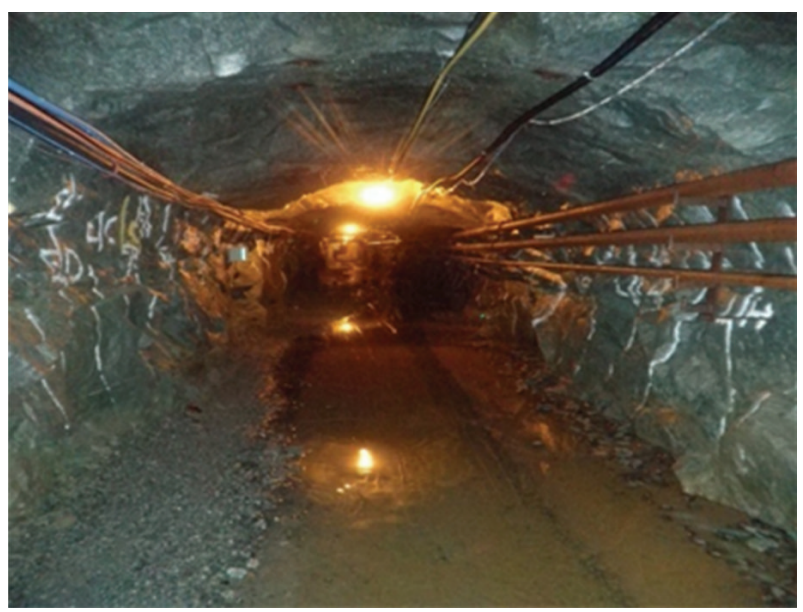

FIgUre 2: Photography of a gallery in CANMET mine at level 70.

inherent ability to sweep over a wide-range frequency band (10 MHz-40 GHz for Agilent E8363B) while maintaining a high receiver sensitivity. For each point considered, the VNA was used to measure and record the complex frequency response, the $S_{21}$ parameter, over a $7 \mathrm{GHz}$ bandwidth (BW) centered on $6.5 \mathrm{GHz}$.

The measurements were taken with the transmitting antenna at a fixed position while the receiver was moved along the gallery. For each position, a specially constructed grid was used for measurement at nine horizontally spaced points around the nominal receiver position. The distance between spatial points is $1 \mathrm{~cm}$, allowing us to avoid the correlation between signals at very close frequencies. In all measurements, the heights of transmitting and receiving antennas are maintained at $1.5 \mathrm{~m}$ in the same horizontal plane, and the channel is kept stationary by ensuring there 


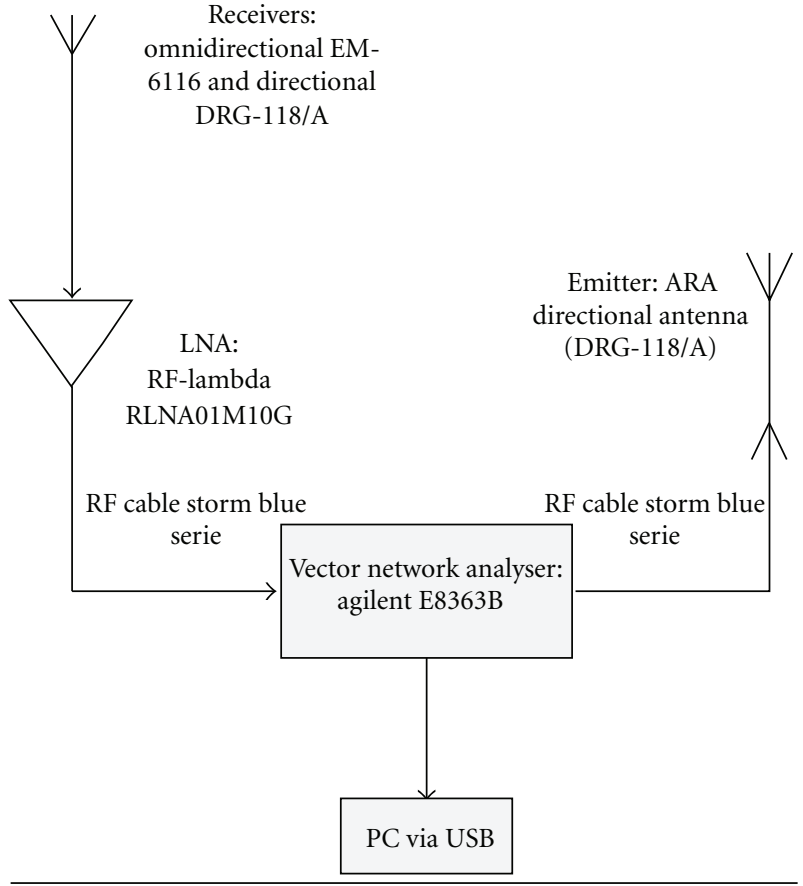

FIGURE 3: Schematic of the experimental protocol.

is no movement in the surrounding environment. To ensure alignment, a laser was used to maintain the same level.

At port 1, a signal was emitted, and at the reception, the receiving antenna was connected to the other port of the VNA with a coaxial cable, a few meters long. The complex channel transfer function is given by the $S_{21}$ parameter $[3,11,12]$.

An ARA directional antenna (DRG-118/A) was used at the emitter. At the receiver, two types of antenna were used. An omnidirectional (EM-6116) and directional DRG-118/A have been used. The EM-6116 antenna is vertically polarized, covering the frequency range from 2 to $10 \mathrm{GHz}$. The DRG118/A antenna has a linear polarization, and the gain varies from 9.1 to $12.2 \mathrm{dBi}$. The $3 \mathrm{~dB}$ beamwidth is $35^{\circ}$ and $20^{\circ}$ for the E-plane and H-plane, respectively. These antennas had an almost flat frequency response from $2 \mathrm{GHz}$ up to $10 \mathrm{GHz}$. The LNA was RLNA01M10G, a product of RF-Lambda. It covers a frequency range from 0.1 to $18 \mathrm{GHz}$, and noise figure is stable along the frequency range. The gain is equal to $30 \mathrm{~dB}$.

In order to be able to subtract the possible antenna effects in the measurements, a reference case was considered by measuring the frequency response of the two antennas in the first meter and then subtracted from the other measure. Amplifiers were not used during the measurements because the distance between the transmitter and the receiver was only a few meters $(10 \mathrm{~m})$. The RF cable length was very short to avoid unwanted effects in the channel characterization. Figure 3 presents the schematic diagram of channel measurement system.

The transmitting port of the VNA swept 16001 discrete frequencies ranged from $3 \mathrm{GHz}$ to $10 \mathrm{GHz}$ uniformly distributed over the bandwidth, and the receiving port measured the magnitude and the phase of each frequency com-

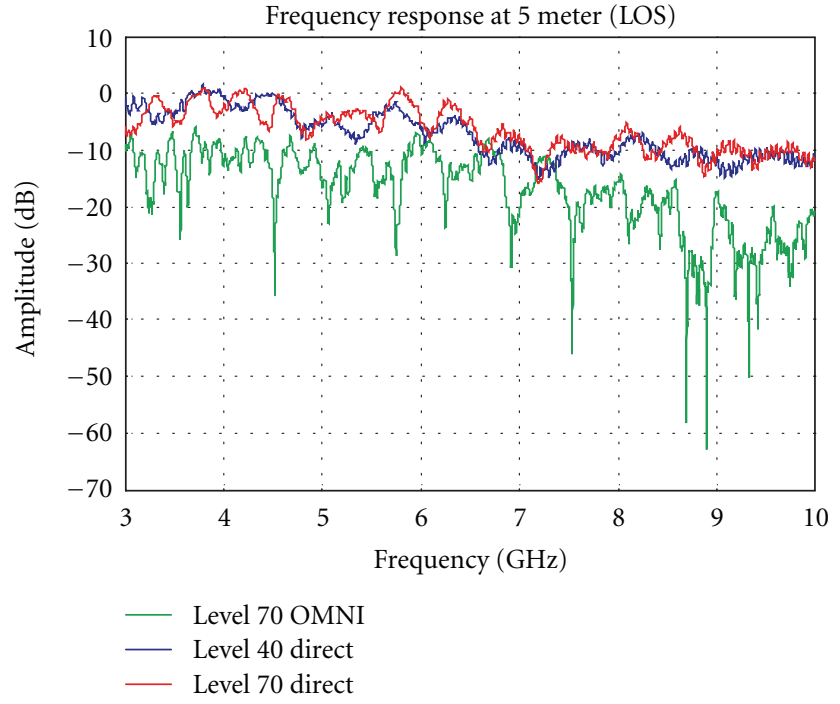

FIgURE 4: Amplitude of frequency response at 5 meter.

ponent. Figure 4 shows a measured complex channel transfer function obtained with the Vector Network Analyzer.

The frequency span of $500 \mathrm{kHz}$ has been chosen narrow enough so that diffraction coefficients and the dielectric constants can be considered constant within the bandwidth of $7 \mathrm{GHz}$ [5]. At each distance between the transmitter and the receiver, the channel transfer function was measured 10 times, in order to reduce the effects of random noise on the measurements, and then stored in a computer hard drive via a USB interface.

Theoretically, the time resolution of the $7 \mathrm{GHz}$ bandwidth is about 143 ps. In practice, due to the use of windowing, the time resolution is estimated to be 2 /bandwidth and the sweeping time of the VNA is decreased to validate the quasi-static assumption of the channel. The frequency resolution of $437.5 \mathrm{kHz}$ gives maximum delay range of $2 \mu$ s corresponding to $600 \mathrm{~m}$ maximum length path which is more than enough for the maximum distance (10 $\mathrm{m}$ ) separating $\mathrm{Tx}$ and $\mathrm{Rx}$, antenna of our measurement setup.

\section{Results}

Whatever are the measurements taken in the time or frequency domain, a channel response has a finite bandwidth that is determined by either the instrument or the measurement process. The result is equivalent to convolving the true channel impulse response with a sinc function whose duration is inversely proportional to the bandwidth of the measurement. Before processing the measured channel impulse responses, the effects of the finite bandwidth either by deconvolution or windowing have to be removed. A windowing was applied to the channel frequency responses in order to suppress dispersion of energy into adjacent delay bins. The channel frequency responses are then converted into complex baseband channel impulse responses by applying an inverse fast Fourier transform (IFFT). Figure 4 presents 
TABLE 1: Frequency decaying factor.

\begin{tabular}{lcc}
\hline & $\delta$ & \\
\hline & $\mathrm{N}-40$ & $\mathrm{~N}-70$ \\
\hline LOS & 1.58 & 1.569 \\
NLOS & 0.314 & 0.534 \\
\hline
\end{tabular}

a frequency response measured at a distance of 5 meter with different antennas (omnidirectional and directive).

The frequency responses at 5 meter have high values due to antennas and LNA gain.

The differences between the frequency responses of omnidirectional antenna and directives antennas are easily noticed (about $10 \mathrm{~dB}$ difference). This difference is explained by the antenna gain $(1 \mathrm{~dB}$ and $12 \mathrm{~dB})$.

3.1. Large-Scale Fading and Path Loss and Shadowing Results. The path loss of the channel represents the attenuation a signal undergoes when transmitted through the medium and is an important parameter in the design of wireless communication systems regarding the coverage. A reference measurement is performed at a distance of $d_{0}$ from the transmitter.

For UWB systems, the path loss modeling can be simplified by assuming that the frequency and the distance dependence can be treated independently of each other:

$$
\operatorname{PL}(d, f)=\operatorname{PL}(d) \cdot \operatorname{PL}(f) .
$$

In frequency analysis, two parameters are often used: the frequency range which is determined by the measured range, and the frequency decaying factor $\delta$. Several works $[13,14]$ have showed that the frequency dependency of the path loss is characterized by this factor. The model of path loss frequency dependence mainly used is shown to be as follows.

The two models were used to fit the variations of the path loss as a function of frequency. Using nonlinear least square curve fitting with the measurements data, as shown in Figure 11 values of $\delta$ are presented in Table 1.

These values are almost equal, and it can be concluded that the geometry of the gallery does not affect the frequency decaying factor.

Using the log-normal shadowing assumption, the path loss exponent, $n$, is related to the receiver power at distance $d$ by

$$
\mathrm{PL}_{\mathrm{dB}}(d)=\mathrm{PL}_{0}\left(d_{0}\right)+10 n \log _{10}\left(\frac{d}{d_{0}}\right)+X_{\sigma},
$$

where $d_{0}$ is the reference distance; in our case, $\mathrm{PL}_{0}\left(d_{0}\right)$ is the interception point and $X_{\sigma}$ modeling a shadowing effect is a zero-mean Gaussian distributed random variable in $\mathrm{dB}$ with the standard deviation. Our measurements were taken in LOS and NLOS. In LOS case, the measurements were made between $1 \mathrm{~m}$ and $10 \mathrm{~m}$ and in NLOS case it was between $1 \mathrm{~m}$ and $10 \mathrm{~m}$, with an interval of $1 \mathrm{~m}$. The results obtained are presented in Figures 5 and 6.

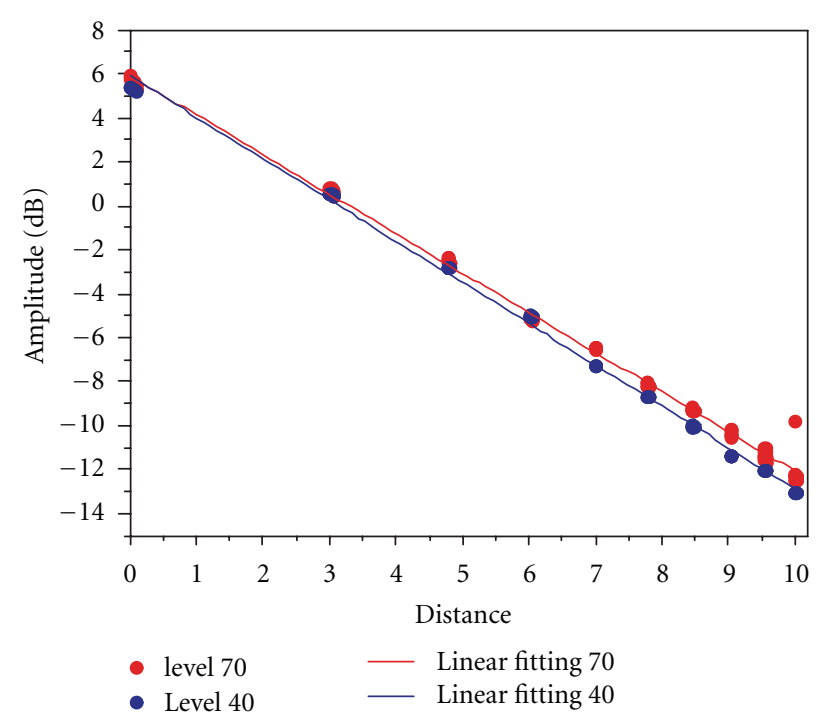

Figure 5: Path loss versus distance (LOS).

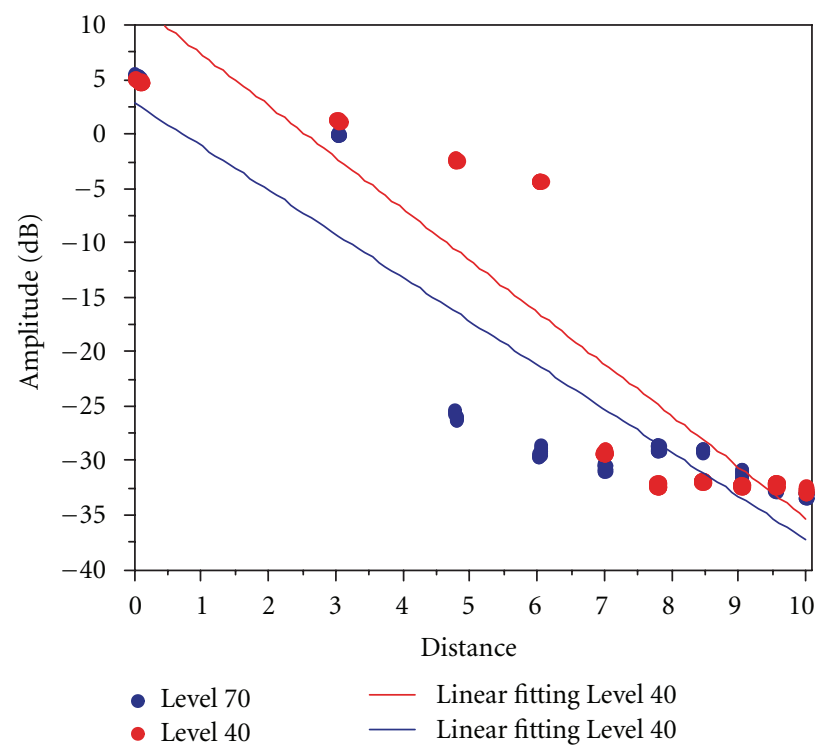

Figure 6: Path loss versus distance (NLOS).

TABLe 2: Path loss exponents and Standards deviations in LOS case.

\begin{tabular}{lcc}
\hline LOS & $\mathrm{N}-70$ & $\mathrm{~N}-40$ \\
\hline$n$ & 1.80 & 1.87
\end{tabular}

TABle 3: Path loss exponents and standards deviations in NLOS case.

\begin{tabular}{lcc}
\hline NLOS & $\mathrm{N}-70$ & $\mathrm{~N}-40$ \\
\hline$n$ & 4.01 & 4.74 \\
\hline
\end{tabular}

Tables 2 and 3 present the path loss exponent $n$ for different configurations.

With the same antenna at level 70 and at level 40, the path loss values are close and there is only a small difference 
between them, probably due to the width of the gallery. The path loss exponent in LOS case is equal to 1.80 at level 70 and 1.87 at level 40 . They are comparable to the to free space path loss exponent $n=2$.

The results of path loss exponent values are due to multipath signal addition and they are similar to those observed in [3] for indoor environments.

In NLOS, the difference is due to the configuration of the gallery. At level 70, there are also some puddles of water. The results obtained show us that the path loss is independent from the depth of the gallery.

3.2. Small-Scale Fading and Delay Spreading Results. Delay spreading parameters highlight the temporal distribution of power relative to the first arriving components. Delay spreads restrict the transmitted data rates and could limit the capacity of the system when multiuser systems are considered. The arrival delay of UWB signal can be presented as the ratio of the average arrival delay to the spread arrival time.

The most appropriate method to describe the rapid fluctuations of amplitude, phase, and multipath delay propagation of a UWB signal is the statistical characterization of the channel impulse response. The number of multipath in an underground environment is more important due to the reflection and scattering from the ground, roof, and surrounding rough walls. The best parameter that allows us to measure multipath is the root mean square delay spread. This parameter determines the frequency selectivity of channel, which degrades the performance of digital communication systems over radio channels. The RMS delay spread also limits the maximum data transmission rate that can be transmitted by the channel. Without using diversity or equalization, the RMS delay spread is inversely proportional to the maximum usable data rate of the channel.

The formulation of time dispersion parameters is given in [15]

$$
\tau_{\mathrm{rms}}=\sqrt{\overline{\tau^{2}}-(\bar{\tau})^{2}}
$$

where $\bar{\tau}$ and $\overline{\tau^{2}}$ are the mean excess delay and the second moment of the PDP, respectively. We can compute the mean excess delay as

$$
\bar{\tau}=\frac{\sum_{k} a_{k}^{2} \tau_{k}}{\sum_{k} a_{k}^{2}}=\frac{\sum_{k} P\left(\tau_{k}\right) \tau_{k}}{\sum_{k} P\left(\tau_{k}\right)},
$$

where $a_{k}, P\left(\tau_{k}\right)$, and $\tau_{k}$ are the gain, power, and delay of the $k$ th path, respectively.

To avoid the effect of noise on the statistics of multipath arrival times, a threshold of $25 \mathrm{~dB}$ below the strongest path was chosen in LOS and NLOS cases. With these assumptions, the root mean square delay spread has been found and the results are depicted in the following tables.

Table 4 shows the RMS delay spread for a directive antenna while Table 5 gives the results for an omnidirectional antenna.

The measured results show that there is no significant advantage gained when using either directive or omnidirectional. This may be explained by the layout of the gallery,
TABLE 4: RMS delay spread in LOS and NLOS cases with directive Antenna.

\begin{tabular}{lcccccc}
\hline $\begin{array}{l}\text { Rms delay spread } \\
\text { (ns) }\end{array}$ & \multicolumn{3}{c}{ Level 70 } & \multicolumn{3}{c}{ Level 40 } \\
& Mean & Std & Max & Mean & Std & Max \\
\hline LOS & 1.762 & 0.727 & 2.837 & 2.0182 & 1.101 & 3.419 \\
NLOS & 4.578 & 2.141 & 7.061 & 5.985 & 4.985 & 11.049 \\
\hline
\end{tabular}

TABLE 5: RMS delay spread in LOS and NLOS cases with omnidirectional antenna.

\begin{tabular}{lccc}
\hline Rms delay spread (ns) & \multicolumn{3}{c}{ Level 70 } \\
& Mean & Std & Max \\
\hline LOS Omni & 1.720 & 0.795 & 2.445 \\
NLOS Omni & 3.761 & 1.740 & 5.675 \\
\hline
\end{tabular}

where multipath components tend to have a limited angle of arrival range due to the guided nature of propagation along the gallery.

To determine the best distribution which represents the experimental result, the Kolmogorov-Smirnov test has been used. The distributions, namely, Rayleigh, Rice, Nakagami, Weibull, and Lognormal chosen are among the most commonly used in wireless communications.

The RMS delay spread at level 40 is plotted and presented in Figure 7 as well as that for level 70 for both types of configuration (LOS, NLOS).

Comparisons with many distributive cumulative functions have been carried out, and the best fit has been found as a lognormal distribution (Figure 8). With these fittings, the K-S test at levels 40 and 70 yields the same result.

In any case, at levels 40 and 70 , the results were the same. Also, the same results were obtained regardless of the measurement configuration in the gallery and whatever the shape of the antenna. Using these results, the coherence bandwidth, which is defined as the range of frequencies over which the channel can be considered flat [15], can be easily computed. The coherence bandwidth at 50 percent is defined by

$$
B_{C}=\frac{1}{5 \tau_{\mathrm{rms}}} .
$$

These results may allow us to determine the maximum bandwidth that can be used for UWB communications in a confined underground without equalization.

3.3. Channel Capacity Analysis. The highest rate of information that can be transmitted through a channel is called the channel capacity. To evaluate the channel capacity, the whole bandwidth $[-B / 2,+B / 2]$ of the UWB channel is divided into a large number of small subchannels with bandwidth $\Delta f=$ $500 \mathrm{kHz}$. From $f$ to $f+\Delta f$, the spectrum can be considered equivalent to a flat fading channel. Thus, by $[16,17]$, the capacity is equivalent to

$$
C=\frac{1}{M} \sum_{k=1}^{M} \log _{2}\left(1+\frac{P\left|H\left(f_{k}\right)\right|^{2}}{N_{0} W}\right),
$$




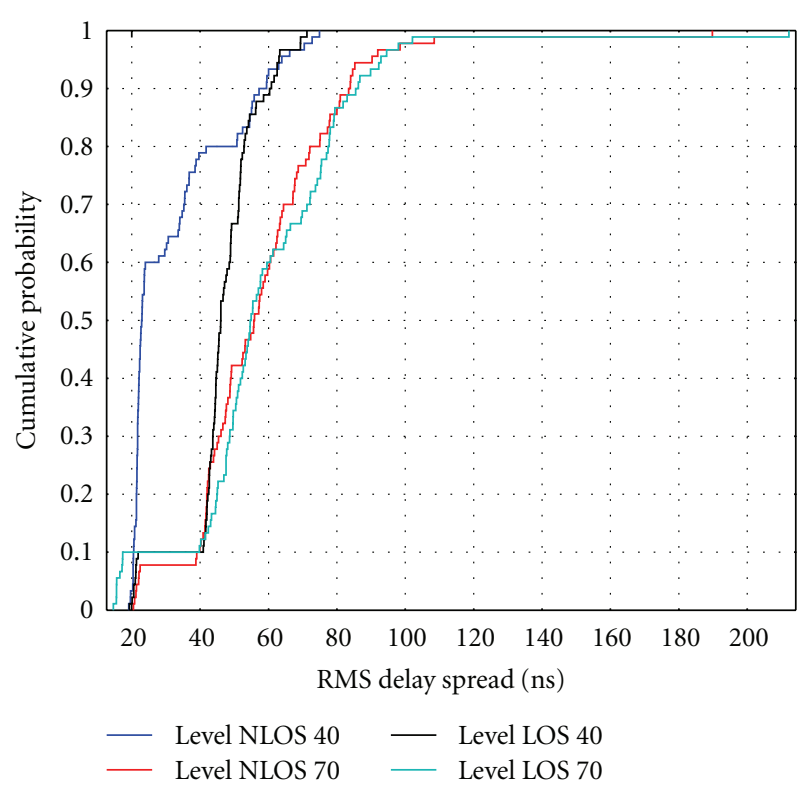

FIgURE 7: RMS delays spread at two levels.

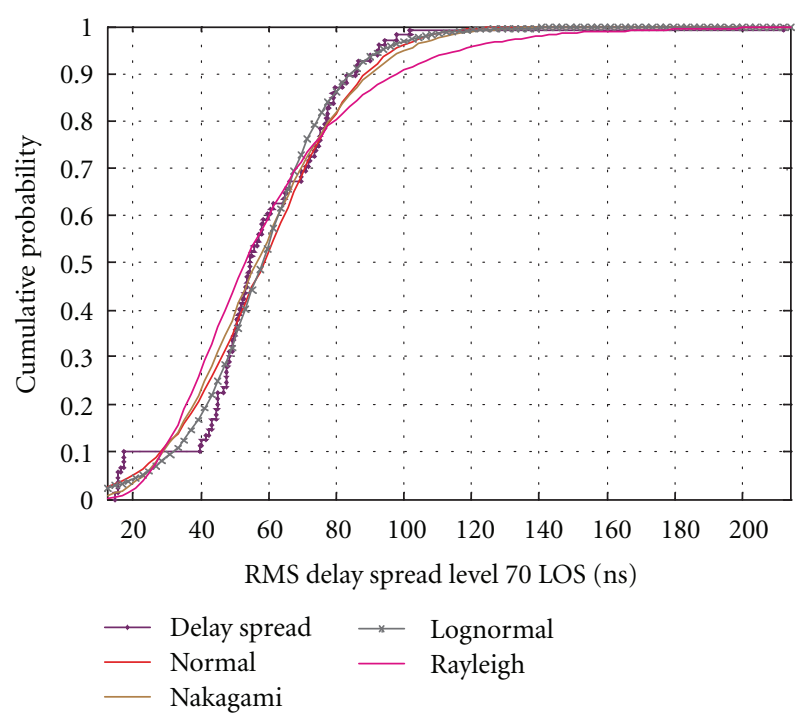

FIGURE 8: Cumulative distribution of RMS at level 70 (LOS).

where $M$ is the number of frequency-domain samples, $|H(f)|$ is the measured frequency response of the channel, $P$ is the transmitted power distributed on the frequency band, $N_{0}$ is a zero-mean white Gaussian noise power spectral density, and $W$ is the bandwidth of the channel.

The channel capacity is computed for each measurement made assuming an SNR of only $10 \mathrm{~dB}$, and results are shown in Figure 9.

The channel capacity is found to decrease with the distance, and also it depends on the configuration of the gallery. The influence of the antenna type on the channel capacity has been investigated. A maximal difference of $1.85 \mathrm{bps} / \mathrm{Hz}$ has been observed between the directive and omnidirectional antenna. This could be explained by the fact that directive antenna receives more power due to its

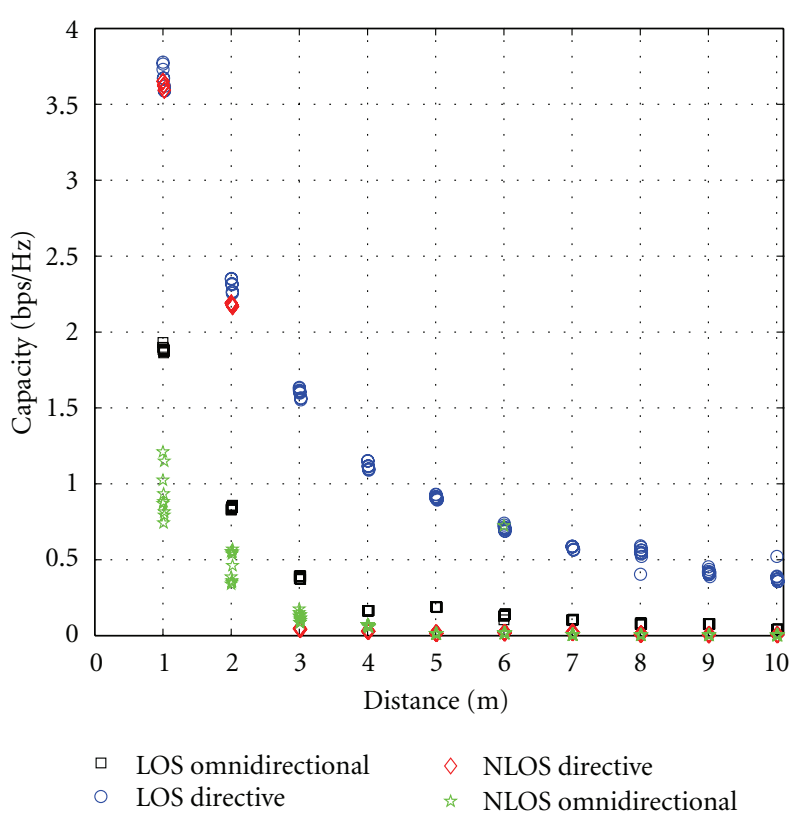

FIGURE 9: Effect of antenna directivity and obstruction on the channel capacity.

radiation pattern diagram. The best results are obtained with LOS configuration and directive antennas. The maximum capacity is then found equal to $3.778 \mathrm{bps} / \mathrm{Hz}$.

Capacity of the channel in different levels of mining gallery has also been computed.

In LOS case with directive antenna, the capacity is almost the same at different levels of mining gallery. In NLOS case, a slight difference is observed, but this is due to the configuration of the mine gallery. This result shows that the UWB channel capacity does not vary with the depth of the gallery (Figure 10).

\section{Conclusion}

The analysis of large- and small-scale parameters has been presented, and fundamental parameters were deduced from these measurements performed in a straight tunnel with a $7 \mathrm{GHz}$ bandwidth $(3 \mathrm{GHz}-10 \mathrm{GHz})$. The results of this study can serve as a unique set of results to characterize propagation in complex media.

These results show that the physical depth of the gallery does not influence the path loss exponent. The small difference observed is mainly due to the width of the gallery. On the other hand, the RMS delay is bound to the depth of the mine and the explanation for it is that there are more absorption and interference at level 70 .

Regarding the small-scale fading, a log-normal distribution fits quite well the amplitude measurements while the phase is better represented using a uniform distribution, with a range of values that decreases with longitudinal distance and increases in frequency.

The channel capacity depends on distance, and the directive antenna gives the best result. It does not depend on the depth of the gallery. 


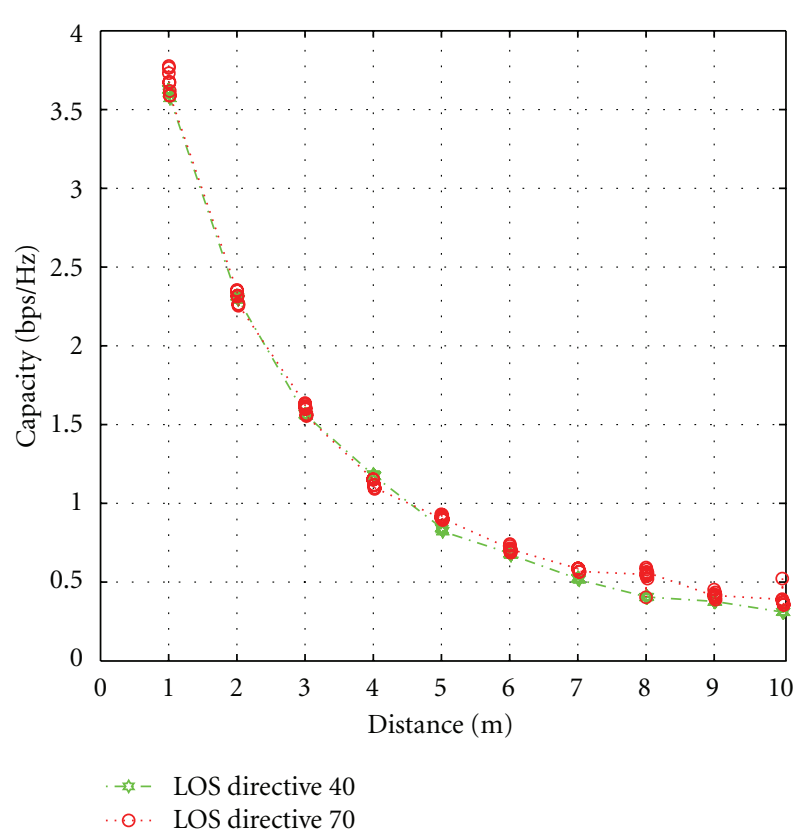

FIGURE 10: Effect of mine corridor size (or physical geometry) on channel capacity.

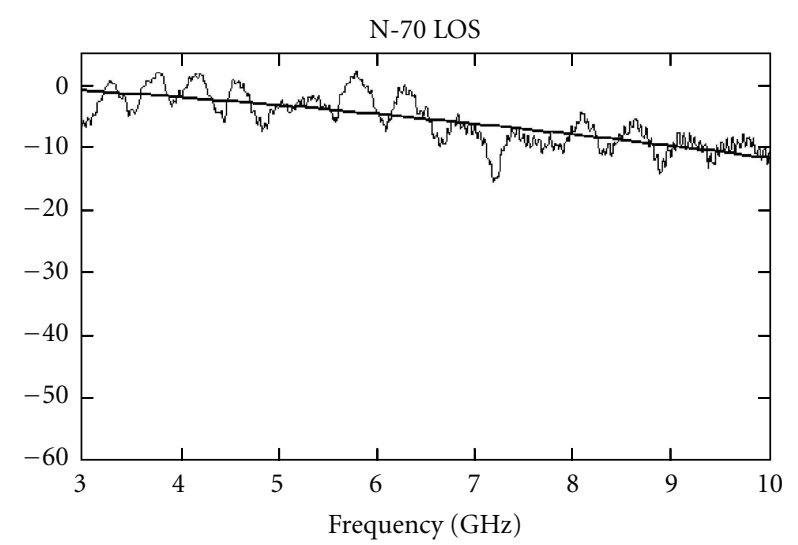

(a)

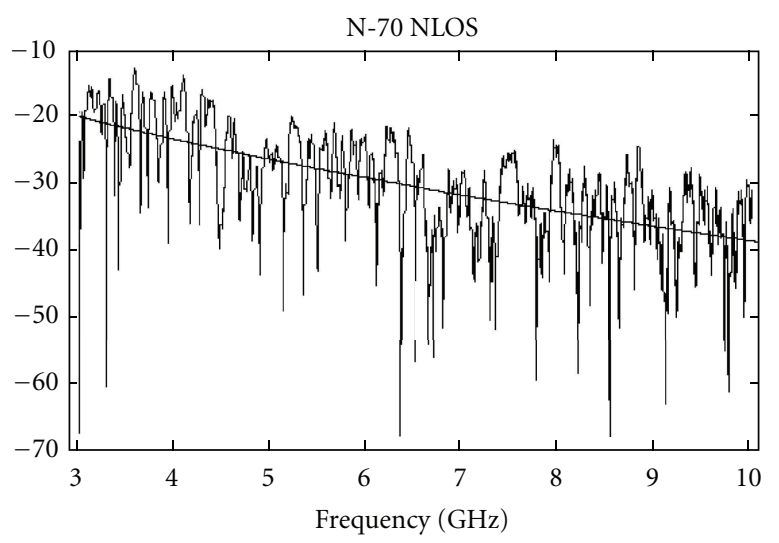

(b)

FIGURE 11: Frequency decaying factor at level 70 in LOS and NLOS.

\section{References}

[1] Federal Communications Commission, First Order and Report: Revision of Part 15 of the Commission's Rules Regarding UWB Transmission Systems, 2002.

[2] A. F. Molisch, D. Cassioli, C. C. Chong et al., "A comprehensive standardized model for ultrawideband propagation channels," IEEE Transactions on Antennas and Propagation, vol. 54, no. 11, part 1, pp. 3151-3166, 2006.

[3] A. F. Molisch, "Ultra-wide-band propagation channels," Proceedings of the IEEE, vol. 97, no. 2, pp. 353-371, 2009.

[4] A. F. Molisch, "Ultrawideband propagation channels-theory, measurement, and modeling," IEEE Transactions on Vehicular Technology, vol. 54, no. 5, pp. 1528-1545, 2005.

[5] C. Nerguizian, C. L. Despins, S. Affès, and M. Djadel, "Radiochannel characterization of an underground mine at $2.4 \mathrm{GHz}$," IEEE Transactions on Wireless Communications, vol. 4, no. 5, pp. 2441-2453, 2005.

[6] M. Boutin, A. Benzakour, C. L. Despins, and S. Affes, "Radio wave characterization and modeling in underground mine tunnels," IEEE Transactions on Antennas and Propagation, vol. 56, no. 2, pp. 540-549, 2008.

[7] A. A. M. Saleh and R. A. Valenzuela, "A statistical model for indoor multipath propagation," IEEE Journal on Selected Areas in Communications, vol. 5, no. 2, pp. 128-137, 1987.

[8] J. M. Molina-García-Pardo, A. Nasr, M. Liénard, and P. Degauque, "Wideband analysis of large scale and small scale fading in tunnels," in Proceedings of the 8th International Conference on Intelligent Transport System Telecommunications (ITST '08), pp. 270-273, Phuket, Thailand, October 2008.

[9] B. M. Donlan, D. R. McKinstry, and R. Michael Buehrer, "The UWB indoor channel: large and small scale modeling," IEEE Transactions on Wireless Communications, vol. 5, no. 10, pp. 2863-2873, 2006.

[10] H. M. A. Al-Tamimi and S. M. Al-Qaraawy, "UWB propagation indoor statistical channel modeling," in Proceedings of the 2nd ISECS International Colloquium on Computing, Communication, Control, and Management (CCCM '09), pp. 379-383, Sanya, China, August 2009.

[11] A. Sani, A. Alomainy, G. Palikaras et al., "Experimental characterization of UWB on-body radio channel in indoor environment considering different antennas," IEEE Transactions on Antennas and Propagation, vol. 58, no. 1, pp. 238-241, 2010.

[12] K. Sarabandi, N. Behdad, A. Nashashibi, M. Casciato, L. Pierce, and F. Wang, "A measurement system for ultrawideband communication channel characterization," IEEE Transactions on Antennas and Propagation, vol. 53, no. 7, pp. 2146-2155, 2005.

[13] A. F. Molisch, B. Kannan, C. C. Chong et al., IEEE 802.15.4a Channel Model-Final Report, IEEE 802.15-04-0662-00-004a, San Antonio, Tex, USA, 2004.

[14] C. C. Chong, Y. Kim, and S. S. Lee, "Statistical characterization of the UWB propagation channel in various types of highvrise apartments," in Proceedings of the IEEE Wireless Communications and Networking Conference (WCNC '05), pp. 944-949, March 2005.

[15] T. S. Rappaport, Wireless Communications Principles and Practice, Prentice-Hall, Englewood Cliffs, NJ, USA, 2002.

[16] D. Tse and P. Viswanath, Fundamentals of Wireless Communication, Cambridge University Press, New York, NY, USA, 2005.

[17] F. Zheng and T. Kaiser, "On the evaluation of channel capacity of UWB indoor wireless systems," IEEE Transactions on Signal Processing, vol. 56, no. 12, pp. 6106-6113, 2008. 

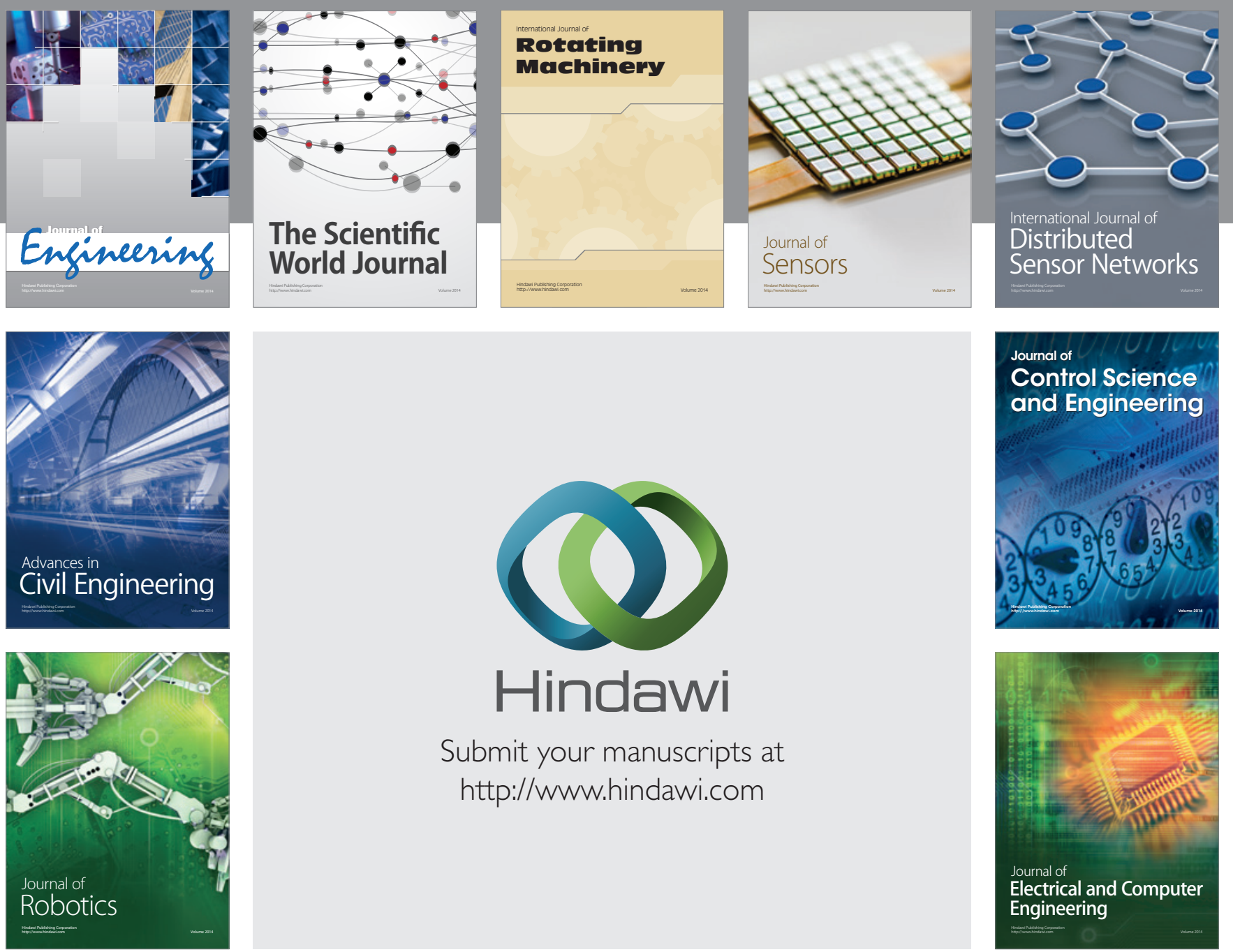

Submit your manuscripts at

http://www.hindawi.com
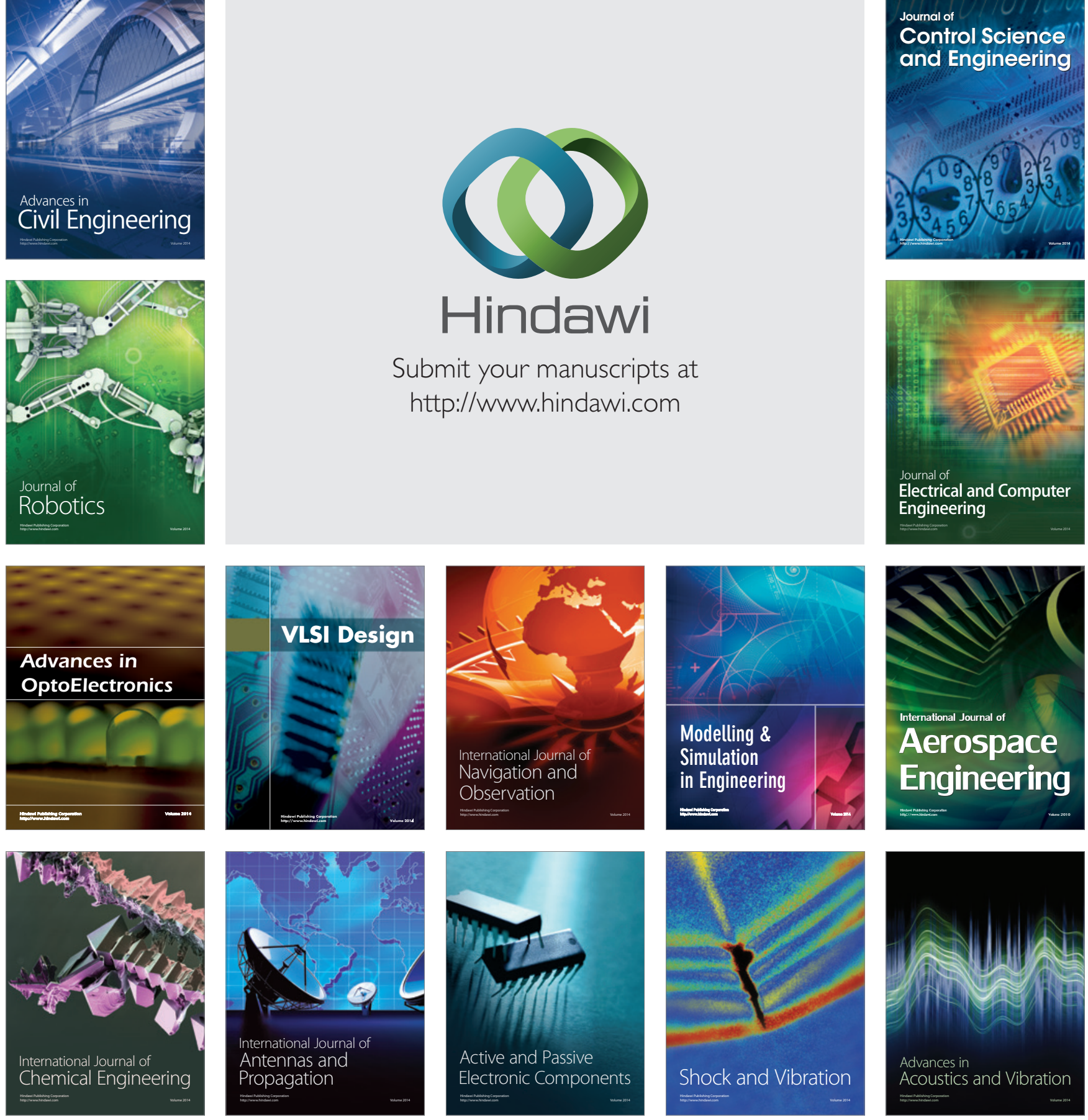